\title{
Strategies of Reducing the Toxicity of Sugar Mill Effluent by Using Biofertilizer Inoculants
}

\author{
Rajesh M., K. Natarajan*, N. Renish \\ Department of Botany, A.V.C.College (Autonomous),Mannampandal-690305 \\ *E-mail address: krishnatarajan31@gmail.com
}

\begin{abstract}
The present investigation has been carried out on Tagetes erecta to search out the effect of sugar mill effluent on growth and yield. In-vivo conditions were set up for the experiment purpose. Pots were filled with soil and treated with different concentrations of sugar mill effluent i.e., 10, 25, 50, 75 and $100 \%$ on morphological parameters of test crop. Results of pot culture experiment showed that at $10 \%$ concentrations of sugar mill effluent proved to better to the crops growth and thereafter the growth may reduce over control. In order to mitigate the effluent toxicity certain Biofertilizers inoculants were mixed to the soil along with the same effluent treatments. The study suggests that the Biofertilizers inoculants along with effluent irrigation proved to be better for plants growth.
\end{abstract}

Keywords: Tagetes erecta; biofertilizer inoculants; plants growth; sugar

\section{INTRODUCTION}

The environment around us, provided by nature, includes the atmosphere, plant and animals. In the olden days, man fully depended upon to nature for his food, shelter and cloth. At that time, the air was fresh, water was pure and land was fertile and there was no trace of pollution in due course of time, man disturbed the nature in order to satisfy his needs of goods and services to lead a luxurious way of life. So, man's relationship with his environment has drastically changed due to a vast increase in his expectations and activities. This resulted in contamination of the air we breath, the water we drink, the food we eat, the medicine we take and the place we work and live, and thus the quality of life has affected and is being affected (Rajesh, 2004).

The sugar industry playing an important role in the economic development of the Indian subcontinent, but the effluents released to produce a high degree of organic pollution in both aquatic and terrestrial ecosystems. They also alter the physico-chemical characteristics of the receiving aquatic bodies and affect aquatic flora and fauna. Sugar factory effluent, when discharged into the environment, poses a serious health hazard to the rural and semi urban population that uses stream and river water for agriculture and domestic purposes, with reports of animal mortality and damage to the agricultural crops in these are due to waste water entering agricultural land (Vijayaragavan et al., 2011). Sugar factory effluent that has 
not been treated properly has an unpleasant odor when released into the environment. Farmers using these effluents for irrigation to reduce water demand have found that plant growth and crop yield were reduced and soil health was compromised. Because sugar industry effluents are commonly used for irrigation, it is essential to determine how crops respond when exposed to industrial effluents. In this regard, efforts have been made to determine the effect of industrial effluents on seed germination of various crops such as maize (Choudhry, et al., 1987), rice (Behra and Mishra., 1982), wheat (Agarwal., et al., 1995) and Green gram (Subramani., et al.,1998)

\section{MATERIALS AND METHODS}

The effluent sample was collected in plastic containers from the place where the effluents are being disposed off by the factory. (N.P.K.R. Ramasamy co-operative sugar mill. Thalainayar, Nagappattinam district of Tamil Nadu).The seeds of African marigold (Tagetes erecta L.) and biofertilizers (Azatobacte sp, Bacillus megaterium and Bacillus mucilaginous) were obtained from market. Pot culture experiment was conducted for a period of 90 days. Red soil and garden soil free from pebbles and sones were filled in pots separately. $10 \mathrm{~kg}$ of soil and sand in the ratio of 3:1 were filled before sowing. Pre - sowing irrigation was given to ensure uniform germination. Irrigation was given at 3 DAS with due care to avoid excess flooding of water. Uniform irrigation was given for 4 times in a month. Five plant samples were randomly collected at regular intervals (30,60 and 90 DAS) and they were used for observations of morphological parameters like root length, shoot length, total leaf area, fresh weight and dry weight of the plant. Pot culture experiments II were conducted with African marigold to know the effect of biofertilizers with $50 \%$ of the effluent concentrations. Two grams of biofertilizers were applied in each pot (soil application).

Table 1. Physico-chemical properties of N.P.K.R. Ramasamy Co-operative sugar mill effluent with their tolerance limits for agricultural irrigation.

\begin{tabular}{|c|c|c|c|}
\hline S.No. & Properties & Raw effluent & $\begin{array}{c}\text { Tolerance limits for } \\
\text { agricultural irrigation } \\
\text { suggested by TNPCB }\end{array}$ \\
\hline 1. & Colour & Dull white & Colourless \\
\hline 2. & Odour & Decaying Molasses smell & - \\
\hline 3. & $\mathrm{pH}$ & 4.03 & $5.5-9.0$ \\
\hline 4. & Electrical conductivity & $4542 \mathrm{Mm}-$ homs & - \\
\hline 5. & Temperature & 34.0 & 40.0 \\
\hline 6. & Acidity & 1272.0 & - \\
\hline 7. & Suspended solids & 214.0 & 100 \\
\hline 8. & Total dissolved solids & 3572.0 & 2100 \\
\hline 9. & Total solids & 3663.0 & 30 \\
\hline 10. & Biological Oxygen Demand & 3266.0 & 250 \\
\hline 11. & Chemical Oxygen Demand & 8263.0 & \\
\hline
\end{tabular}




\begin{tabular}{|c|c|c|c|}
\hline 12. & Chloride & 326.0 & 2.0 \\
\hline 13. & Sulphate & 310.0 & 2.0 \\
\hline 14. & Nitrate & 52.44 & - \\
\hline 15. & Fluoride & 1.63 & 1.0 \\
\hline 16. & Silica & 96.0 & - \\
\hline 17. & Calcium & 122.6 & 1000.0 \\
\hline
\end{tabular}

All parameters except colour, odour, $\mathrm{pH}, \mathrm{EC}$ and Temperature are expressed in $\mathrm{mg} / \mathrm{l}$ TNPCB - Tamil Nadu Pollution Control Board

Table 2. The Morphological parameters of African marigold (Tagetes erecta L.) grown under different concentrations of Sugar mill effluent.

\begin{tabular}{|c|c|c|c|c|c|c|c|c|c|c|c|c|c|c|c|}
\hline \multirow[b]{2}{*}{ 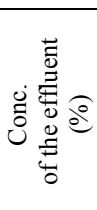 } & \multicolumn{5}{|c|}{$30 \mathrm{DAS}$} & \multicolumn{5}{|c|}{$60 \mathrm{DAS}$} & \multicolumn{5}{|c|}{$90 \mathrm{DAS}$} \\
\hline & 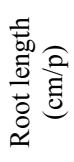 & 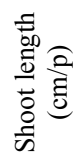 & 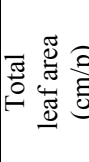 & 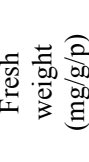 & 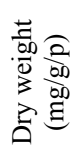 & 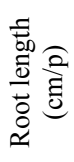 & 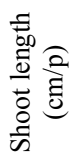 & 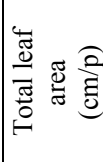 & 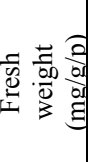 & 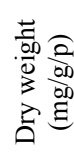 & 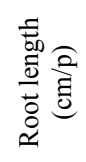 & 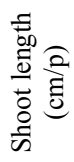 & 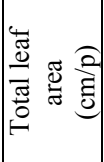 & 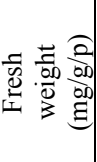 & 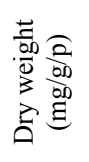 \\
\hline Control & $\stackrel{?}{\stackrel{f}{r}}$ & $\stackrel{\circ}{\stackrel{0}{\circ}}$ & $\begin{array}{l}\stackrel{7}{*} \\
\stackrel{+}{0}\end{array}$ & 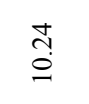 & $\underset{\vec{i}}{\Delta}$ & ֻ̇ & $\begin{array}{c}\infty \\
\substack{\infty \\
\infty \\
i}\end{array}$ & 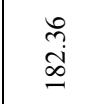 & $\begin{array}{l}\infty \\
\substack{\infty \\
\infty \\
i}\end{array}$ & $\stackrel{7}{\rightarrow}$ & $\stackrel{?}{=}$ & $\begin{array}{l}\stackrel{\infty}{0} \\
\stackrel{0}{0}\end{array}$ & 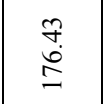 & ণ্ণి & శ్ \\
\hline 5 & 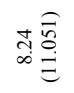 & 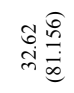 & 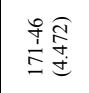 & 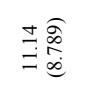 & 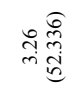 & 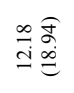 & 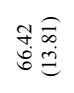 & 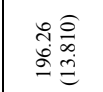 & 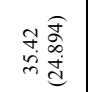 & 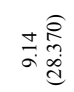 & 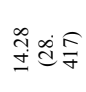 & 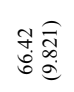 & 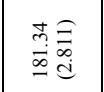 & $\begin{array}{l}\stackrel{\infty}{\vec{g}} \\
\text { 马े }\end{array}$ & $\begin{array}{ll}\infty \\
\infty \\
\infty\end{array}$ \\
\hline 10 & 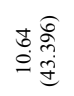 & 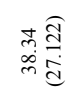 & 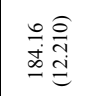 & 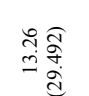 & 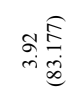 & 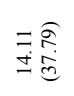 & 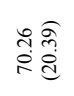 & 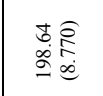 & 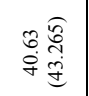 & 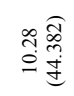 & 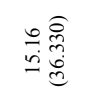 & 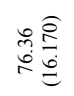 & 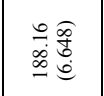 & 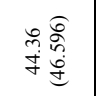 & 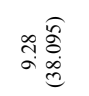 \\
\hline 25 & 旅 & 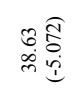 & 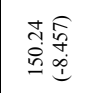 & 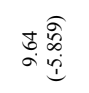 & 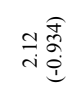 & 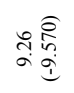 & 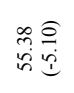 & 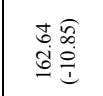 & 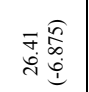 & ชู่ & 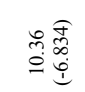 & 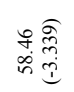 & 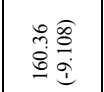 & 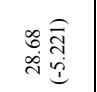 & 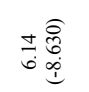 \\
\hline 50 & 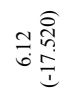 & 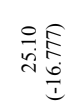 & 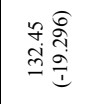 & ব্ণ & 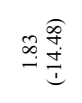 & 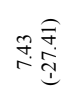 & 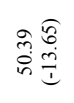 & 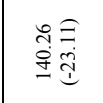 & 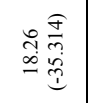 & 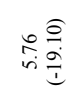 & 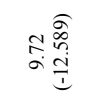 & 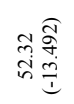 & 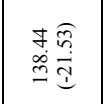 & 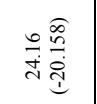 & 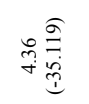 \\
\hline 75 & 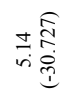 & 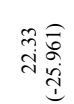 & 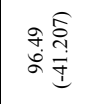 & 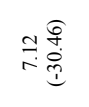 & 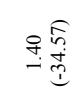 & 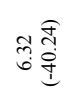 & 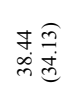 & 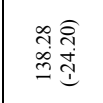 & 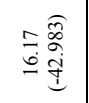 & 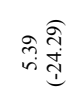 & 㕍 & 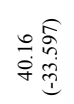 & 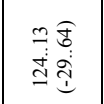 & 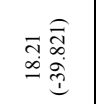 & 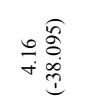 \\
\hline 100 & 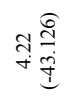 & 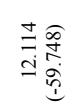 & 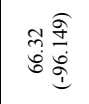 & 告产 & 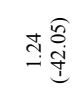 & 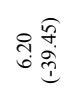 & 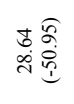 & 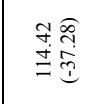 & 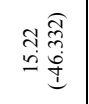 & 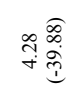 & 羿言 & 荡总 & 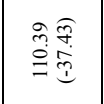 & 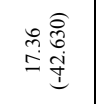 & 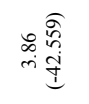 \\
\hline
\end{tabular}

\pm Percentage over control is expressed in paranthesis. 
Table 3. Yield parameters of African Marigold (Tagetes erecta L.) grown under different concentrations of the effluent.

\begin{tabular}{|c|c|c|c|}
\hline $\begin{array}{c}\text { Effluent concentrations in } \\
\text { percentage }\end{array}$ & Number of flowers & $\begin{array}{c}\text { Weight of the flower } \\
\text { (g/flower) }\end{array}$ & $\begin{array}{c}\text { Yield of the flower } \\
\text { (g/pot) }\end{array}$ \\
\hline Control & 62.0 & 10.0 & 550.0 \\
\hline 5 & 69.0 & 12.3 & 600.0 \\
& $(11.290)$ & $(23.00)$ & $(9.090)$ \\
\hline 10 & 75.0 & 18.6 & 750.0 \\
& $(20.967)$ & $(86.00)$ & $(36.363)$ \\
\hline 25 & 48.0 & 9.6 & 400 \\
& $(-22.580)$ & $(-4.00)$ & $(-27.272)$ \\
\hline 50 & 45.0 & 6.2 & 350 \\
& $(-27.419)$ & $(-38.00)$ & 200 \\
\hline 75 & 70.0 & 4.2 & $(-63.636)$ \\
\hline 100 & $(-83.870)$ & $(-58.00)$ & 100 \\
& 6.0 & 2.1 & $(-81.818)$ \\
\hline
\end{tabular}

\pm Percentage over control is expressed in paranthesis.

Table 4. Effect of Biofertilizers on morphological parameters of African marigold (Tagetes erecta L.) grown underthe 50 per cent concentration of the sugar mill effluent.

\begin{tabular}{|c|c|c|c|c|c|c|c|c|c|c|c|c|c|c|c|}
\hline \multirow[b]{2}{*}{ 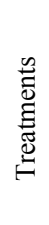 } & \multicolumn{5}{|c|}{30 DAS } & \multicolumn{5}{|c|}{60 DAS } & \multicolumn{5}{|c|}{90 DAS } \\
\hline & 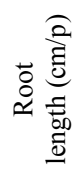 & 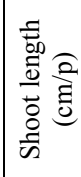 & 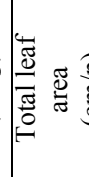 & 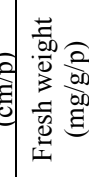 & 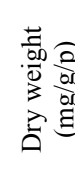 & 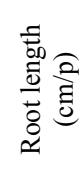 & 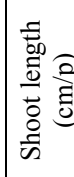 & 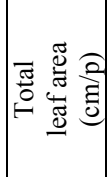 & 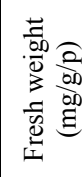 & 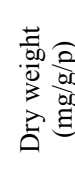 & 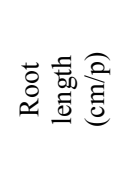 & 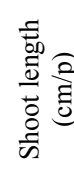 & 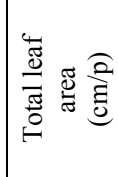 & 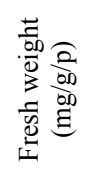 & 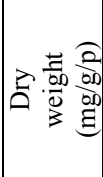 \\
\hline $\mathrm{T} 1$ & $\underset{\infty}{\mathcal{f}}$ & 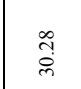 & fै & $\stackrel{\infty}{=}$ & 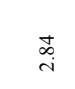 & 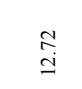 & $\begin{array}{l}\text { הี } \\
\text { है }\end{array}$ & $\begin{array}{l}\text { त̃ } \\
\stackrel{5}{\circ}\end{array}$ & $\stackrel{\infty}{\stackrel{\infty}{0}}$ & 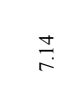 & $\stackrel{\infty}{\stackrel{\infty}{g}}$ & $\frac{\pi}{0}$ & $\begin{array}{l}\underset{+}{+} \\
\stackrel{+}{\infty} \\
\stackrel{\infty}{n}\end{array}$ & $\underset{m}{\stackrel{t}{m}}$ & శై \\
\hline T2 & 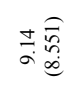 & वृ & 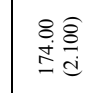 & 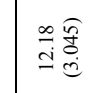 & 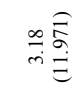 & 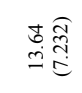 & 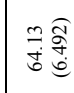 & 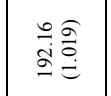 & $\underset{\substack{\infty \\
\infty}}{\widehat{S}}$ & 赵 & 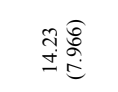 & 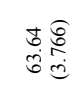 & 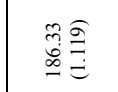 & 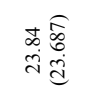 & 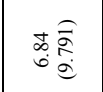 \\
\hline т3 & ه্ & 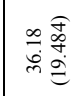 & 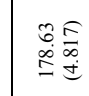 & 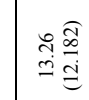 & 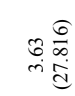 & 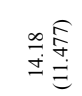 & 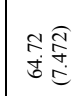 & $\begin{array}{l}\stackrel{0}{\hat{\alpha}} \\
\text { da }\end{array}$ & 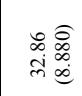 & 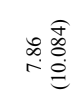 & 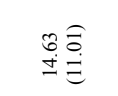 & 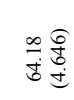 & 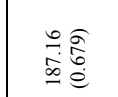 & 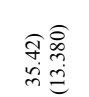 & 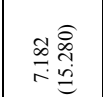 \\
\hline
\end{tabular}




\begin{tabular}{|c|c|c|c|c|c|c|c|c|c|c|c|c|c|c|c|}
\hline $\mathrm{T} 4$ & 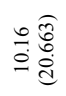 & 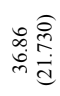 & 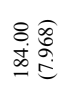 & 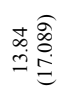 & \& & 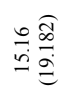 & $\begin{array}{l}m \widehat{\hat{n}} \\
\vec{b}\end{array}$ & 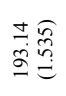 & 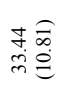 & 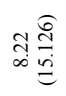 & 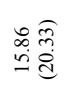 & 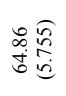 & 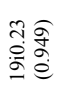 & 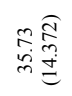 & 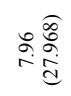 \\
\hline T5 & 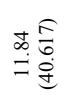 & 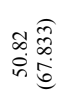 & 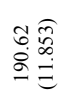 & 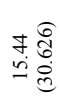 & 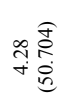 & 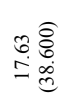 & 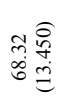 & 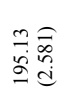 & 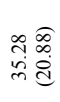 & 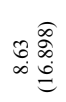 & 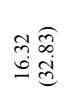 & 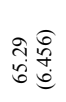 & 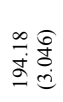 & 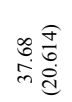 & 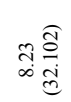 \\
\hline
\end{tabular}

\pm Percentage over control is expressed in paranthesis.
$\overline{\mathrm{T}} 1 \quad$ - Control (distilled water).
T2 - Azatobacter sp.,
T3 - Bacillus megaterium, T4 - Bacillus mucilaginous., T5 - (T2 + T3 + T4)

Table 5. Effect of Biofertilizers on yield parameters of African Marigold (Tagetes erecta L.) grown under the 50 per cent concentration of the sugar mill effluent.

\begin{tabular}{|c|c|c|c|}
\hline Treatments & Number of flowers & Weight of the flowers $(\mathrm{g})$ & Yield of the flowers $(\mathrm{g})$ \\
\hline $\mathrm{T} 1$ & 65.0 & 9.0 & 450.0 \\
\hline \multirow{2}{*}{$\mathrm{T} 2$} & 68.0 & 10.2 & 500.0 \\
& $(4.615)$ & $(13.33)$ & $(11.11)$ \\
\hline \multirow{2}{*}{$\mathrm{T} 3$} & 72.0 & 14.3 & 520.0 \\
& $(10.76)$ & $(58.88)$ & $(15.55)$ \\
\hline \multirow{2}{*}{$\mathrm{T} 4$} & 73.0 & 15.2 & 540.0 \\
& $(12.30)$ & $(68.88)$ & $6000)$ \\
\hline \multirow{2}{*}{ T5 } & 80.0 & 16.2 & $(33.33)$ \\
\hline
\end{tabular}

\pm Percentage over control is expressed in paranthesis.
$\overline{\mathrm{T}} 1 \quad$ - Control (distilled water).
T2 - Azatobacter sp.
T3 - Bacillus megaterium, T4
- Bacillus mucilaginous., T5 - (T2 + T3 + T4)

\section{RESULTS AND DISCUSSION}

Water pollution is referred to addition to water, an excess of material that is harmful to plants, humans, animals and aquatic life or otherwise causes significant deviations from the normal activities or various living communities in or near by water bodies (Pandey, 1992). Everything is polluted today, the rivers, seas, lakes almost all water bodies are polluted by waste coming from various industries. The physico-chemical analyses of sugar mill effluent showed that the effluent was acidic in nature, it contained high amount of suspended solids and dissolved solids resulting in high Biological Oxygen Demand and Chemical Oxygen Demand (Table-1). It also contained more quantities of Chloride, Sulphate, Silica, Sodium, Potassium, Calcium, etc. The similar findings were also reported in sugar mill effluent (Thamizhiniyan et al., 2009: Doke et al., 2011 and Samuel and Muthukaruppan, 2011).

The shoot length and root length were found maximum in the African marigold treated with 10 per cent concentration of the effluent( Table-2 \&3). The lengths decreased gradually with increased concentration of the effluent. The similar observation were made on Blackgram (Ravimycin and Lakshmanachary (1993), Bhendi (Rathinasamy and Lakshminarashimhan, 1998), Raphanus sativus (Vijayaragavan et al., 2011) and Peanut and Green gram. (Siva Santhi and Suja Pandian, 2012). The increase growth rate of the plants is 
due to the absorption of essential elements such as nitrogen, calcium and sodium from the soil treated with effluent (Singh and Mishra, 1987). The inhibitory effect of the effluent at its high concentrations $(25,50,75$ and $100 \%)$ on plant growth might be due to the presence of suspended and dissolved solids, anions and cations in high quantities which exceed the tolerance limits. The total leaf area showed an increasing trend in plants treated with low concentrations (upto 10\%) of the effluent and decreased in those treated with high concentration. The similar trend was observed in Hordeum vulgare treated with Carbonaceous sugar mill effluent (Kumar, 2000). The growth promoting effect of nitrogen, magnesium, potassium and calcium present in optimum quantities in the lower concentration of the effluent might be reason for the increase in number of leaves and leaf area. The same elements in their excessive quantity present in the higher concentrations of the establishment might have inhibited the growth of plants by interfering with water absorption and metabolic process (Rani and Srivastava, 1990).

The fresh weight and dry weight of African marigold increased with the age of the plants in all concentration (Table $2 \& 3$ ). Off all concentration, the dry weight of the organs increased upto 10 per cent and decreased thereafter. The similar results were also found in Raphanus sativus (Vijayaragavan et al., 2011). The increase in all the morphological parameters at low concentrations could be attributed to the uptake nitrogen, calcium, sodium, and chloride from effluents by plants via soil media (Thamizhiniyan et al., 2009). The reduction in the fresh weight and dry weight at high concentrations of effluent is an indicator of the inhibitory effect of the effluent on the synthesis of protein and photosynthetic activity due to carbonaceous sugar mill effluent on Hardeum Vulgar (Arindam, 1996) changes in the chlorophyll 'a', chlorophyll 'b', total chlorophyll and carotenoid at different stages of African marigold grown under the influence of sugar mill effluent were estimated. The pigment contents showed on increasing trend with the increase in the age of plant upto 60 days and then decreased at harvest state.

The Morphological parameters such as, Root length, shoot length, total leaf to area, fresh weight and dry weight were increased at 50 per cent effluent with combined application of biofertilizers in various days $(30,60$ and 90 DAS) than that of control and other biofertilizers alone( Table-4). The same trend was observed by Ravindran et al., (2007) .The yield parameters such a number of flowers, weight of a flower and yield of flower were increased in 50 per cent effluent concentration with combined application of Biofertilizers when compared with control and other Biofertilizers alone(Table-5). The same trend was observed in maize (Rajeswar, 2010).

Since, there was increase in growth, biochemical and yield at low concentration of effluent. It is recommended that the sugar mill effluent can be used for irrigation after diluted properly, Hence the effluent at 10 per cent level could serve as a good liquid fertilizer. It already established that the application of these organisms (Biofertilizers) in the form of bio inoculants showed beneficial effect in crops and flowering plants. Hence, it is concluded that the said microbial inoculants could be formulated and delivered as bio inoculants to farmers.

\section{Acknowledgement}

The authors are thankful to the UGC for sanctioning the grantfor conducting the research work. 


\section{References}

[1] Arindam, K. 1996. Action of industrial effluents on plant genetic system. Ph.D. Thesis, L.N. Mithila University, Durbhanga, Bihar.

[2] Agarwal SR, Chaturvedi C, Chaturvedi C. 1995. Effect of industrial effluents of a paper and sugar mill on the germination of wheat (Triticum aestivum). J. Livin. Wld., 2: $16-19$.

[3] Behera, B.K. and B.N. Mishra, 1982. Analysis of the effect of industrialeffluents on growth and development of rice seedlings. Environ. Res., 28: 10-20.

[4] Choudhury SK, Jha AN, Srivastava DK,1982. Effect of paper mill effluent on seed germination and seedling growth in maize. Environ. Ecol., 5: 285-287.

[5] Doke, K.M., M.E., Khan, J. Rapolu and A. Shakib, 2011. Physico-Chemical analysis of sugar industry effluent and its effects on seed germination of Vigna arcularis, Vigna cylindrical and Sorghum celnum. Ann. Environ. Sci., 5: 7-11.

[6] Kumar.A.2000. Carbonaceous sugar mill effluent retards growth and yield of Hordeum vulgare IB65. Ad. Plant Sci., 13(11): 93 - 96.

[7] Pandey, V. 1992. Water and marine pollution In: Encyclopaedic dictionary of environmental pollution. Himalaya Publishing House, Bombay, 2: 266.

[8] Rajesh, M. 2004. Screening of paddy cultivars for tolerance of sugar mill effluent irrigation.

[9] Ph.D. Thesis.

[10] Rajeshwar, M.M.A., A. Khan, 2010. Effect of biofertilizers on crop yield and soil available nutrient of rice and maize in altisols of Nagarjuna sugar left canal command area of Andhra Pradesh, India, An Asian.J.Soil. Sci., 5(1): 200-203.

[11] Rani, R.and M.M. Srivastava, 1990. Ecophysiogical response of Pisum sativum and Citrus maxima to distillery effluents. J. Ecol., and Environ. Sci., 16(2\&3): 125-132.

[12] Rathinasamy, A and C.R. Lakshminarashimhan, 1998. Effect of sugar factory effluent on growth, yield and quality of bhendi-var. PKM-1. Madras Agric. J., 85 (7-9): 403405.

[13] Ravimycin, T., and A.S. Lakshmanachary, 1993. Impact of sugar factory effluent on seed germination, seedling growth and yield of blackgram (vigna murgo (L.) Hepper) Bio-Science Research Bulletin, 9(1-2): 17-22.

[14] Ravindran, K.C., K. Venkatesan, T. Balasubramanian and V. Balakrishanan, 2007. Effect of halophytic compost along with farm yard manure and phosphobacteria on growth characteristics of Ararchis hypogaca L., Science of the total Environ., 333341.

[15] Samuel, S., and S.M.Muthukkaruppan, 2011. Physico - Chemical Analysis of sugar Mill Effluent, Contaminated soil and its effect on seed germination of paddy (Oryza sativa L). International Journal of Pharamaceutical \& Biological Archives, 2(5): 1469-1472. 
[16] Singh, K.K. and L.C.Mishra, 1987. Effect of fertilizer factory effluent on soil and crop productivity. Water, Air and Soil Pollution, 33: 309 - 320.

[17] Siva Santhi, K and R. Suja Pandian, 2012. Effect of sugar mill effluent on seed germination of peanut (Arachis hypogaea) and green gram (Vigna radiata). Int. J. Pharm. Chem. Sci., 1: 804-806.

[18] Subramani, A., S. Saravanan, P. Sundaramoorthy and A.S. Lakshmanachary, 1998. Impact of fertilizer factory effluent on the morphometrical and biochemical changes of cowpea (Vigna unguiculata L.) Walp. Ad. Plant Sci., 11(1): 137-141.

[19] Thamizhiniyan, P., Sivakumar, P.V., Lenin M. and Sivaraman. M (2009) Sugar Mill Effluent Toxicity in Crop plants ,J. Phytol. 1: 68-74.

[20] Vijayaragavan, M., C. Prabhahar, J. Sureshkumar.,. A. Natarajan, P. Vijayarengan and S. Sharavanan, 2011. Soil irrigation effect of sugar mill effluent on changes of growth and biochemical contents of Raphanus sativus L. Curr. Bot., 2: 09-13. 